\title{
Le chevalier Hercule de l'Ovide moralisé au Livre de la mutacion de fortune de Christine de Pizan
}

\section{Liliane Dulac}

\section{(2) OpenEdition \\ 1 Journals}

Édition électronique

URL : https://journals.openedition.org/crm/68

DOI : $10.4000 / \mathrm{crm} .68$

ISSN : 1955-2424

Éditeur

Honoré Champion

Édition imprimée

Date de publication : 10 décembre 2002

ISSN : 1272-9752

Référence électronique

Liliane Dulac, «Le chevalier Hercule de l'Ovide moralisé au Livre de la mutacion de fortune de Christine de Pizan », Cahiers de recherches médiévales [En ligne], 9 | 2002, mis en ligne le 03 septembre 2007, consulté le 15 décembre 2022. URL : http://journals.openedition.org/crm/68 ; DOI : https://doi.org/ $10.4000 / \mathrm{crm} .68$

Ce document a été généré automatiquement le 15 décembre 2022.

Tous droits réservés 


\title{
Le chevalier Hercule de l'Ovide moralisé au Livre de la mutacion de fortune de Christine de Pizan
}

\author{
Liliane Dulac
}

à Glynis Cropp

L'éditrice de la Mutacion de Fortune ${ }^{1}$ observe que c'est à propos du personnage d'Hercule que Christine de Pizan a, dans cette œuvre, fait l'emprunt le plus important à l'ovide moralisé $e^{2}$ qui, avec l'Histoire ancienne jusqu'à César ${ }^{3}$, constitue une des sources principales de cet immense poème en forme d'histoire universelle. Deux ans avant l'achèvement de la Mutacion (1403), dans son Epistre Othea ${ }^{4}$, premier ouvrage de sa "nouvelle manière " vouée à la morale, à l'histoire et à la politique, un épisode du mythe d'Hercule également tiré de l'Ovide moralisé fournit le motif narratif de deux des cent chapitres, ou "séquences ", qui dessinent la figure du bon chevalier. Il peut donc paraitre judicieux de choisir l'exemple de ce personnage, par ailleurs un des plus complexes de la tradition mythologique antique, pour apprécier la manière dont Christine utilise la matière ovidienne: ou du moins celle qu'elle pouvait trouver dans l'énorme Ovide français en vers, qui tout en suivant assez fidèlement les Métamorphoses ${ }^{5}$, y ajoute non seulement des commentaires moraux, historiques et surtout allégoriques, mais aussi des épisodes entiers ${ }^{6}$. Pour autant, notre propos restera des plus modestes : nous nous en tiendrons au seul personnage d'Hercule et aux deux épisodes que Christine a empruntés à l'Ovide moralisé, la descente aux enfers (sujet des séquences 3 et 27 d'Othea), et d'autre part la succession d'événements qui, en rendant effective la vengeance du centaure Nessus, conduiront le héros à choisir de mourir sur le bûcher (Mutacion, $\mathrm{VI}^{\mathrm{e}}$ partie). Ces fables, qui dans les deux œuvres reçoivent un traitement très différent, ne comportent ni implications autobiographiques (contrairement à celles qui, dans la Mutacion, accompagnent la métamorphose de Christine en homme et en écrivain ${ }^{7}$ ), ni allusions politiques précises (comparables à celles qu'on peut déceler sous certaines «couvertures " poétiques, par exemple à propos du personnage d'Actéon ${ }^{8}$ ), et elles ne traduisent pas non plus un engagement personnel ${ }^{9}$. Posons donc d'emblée que 
l'exemple choisi est à priori sans relief particulier, mais peut par là même offrir un terrain d'observation à qui tente d'analyser les modalités du travail ordinairement effectué par Christine.

Notons que nous ne prendrons pas en considération, sinon de façon marginale, deux autres épisodes de la vie d'Hercule évoqués dans ces œuvres. C'est tout d'abord l'histoire de Laomédon, père de Priam, qui offense les Argonautes lorsqu'ils abordent dans son pays, ce qui entraînera la destruction de la première Troie par les Grecs sous le commandement d'Hercule: elle figure dans deux séquences d'Othea (37 et 66) et prélude dans la Mutacion aux récits des guerres de Troie. Mais outre que cette fable n'est qu'en partie redevable à l'Ovide moralisé, elle a pour personnage central Laomédon. Quant à l'histoire de l'expédition qu'Hercule conduit en compagnie de Thésée contre les Amazones, afin de protéger la Grèce de la menace qu'elles font peser sur leur pays, elle contribue dès le début de la $\mathrm{VI}^{\mathrm{e}}$ partie de la Mutacion à camper la figure d'un preux aussi magnanime que vaillant et dévoué au bien public: mais l'épisode est emprunté à l'Histoire ancienne ${ }^{10}$ et sort donc du champ de nos observations.

3 Nous ne saurions rappeler que très sommairement dans quel contexte se situe le travail de Christine sur le point précis que nous avons choisi d'étudier : c'est-à-dire l'étonnante fortune que connaît le mythe d'Hercule, tout particulièrement dans les derniers siècles $\mathrm{du}$ Moyen Âge et jusqu'en pleine Renaissance ${ }^{11}$. Alors qu'aux premiers temps de l'apologétique chrétienne, un auteur comme Lactance ne voulait voir dans le fils d'Alcmène qu'un guerrier immoral, plein de fureur et de folie ${ }^{12}$, une «aventure » de plusieurs siècles a fait de lui un symbole chrétien non seulement de la vaillance, mais de la force morale et des plus hautes vertus - ce qu'il est déjà chez Boèce ${ }^{13}-$, puis une figure de Dieu, ou de son incarnation sur terre. Ainsi dans l'Ovide moralisé, la fable d'Hercule tuant le centaure Nessus représente Dieu, «le biau combateour / Et le glorieus vainqueour» qui triomphe du Diable (livre IX, vv. 475-476), et les épreuves qu'il subit sont assimilées à la vie terrestre du Christ. Sur ce terrain, nombreux seront les parallèles entre Hercule et le Sauveur ${ }^{14}$ : tous deux, ils ont combattu le Mal, sont descendus en enfer et ont péri dans la douleur, avant de connaître l'apothéose finale. D'autres applications du mythe auront à la fin du Moyen Âge une dimension politique, notamment quand Hercule incarnera la puissance princière ou royale, jusqu'à ce que François $\mathrm{I}^{\mathrm{er}}$ se fasse représenter sous les traits du héros antique ${ }^{15}$. Bien que de façon discrète, Christine elle-même, dans la Mutacion, ne présente pas Hercule comme un simple chevalier:

Herculès roy ert couronné

De grant paÿs et de regné.

Belle Yole il prist a femme,

Fille d'un roy, moult haute dame. (vv. 13919-13922)

4 Ainsi l'utilisation particulière qu'elle fait des matériaux fournis par l'Ovide moralisé ne peut être isolée du mouvement général qui, de son temps, entraîne le développement de différentes mises en œuvre du mythe et l'accroissement de sa popularité : on ne saurait ignorer tout à fait les intertextes qui à cet égard pouvaient entrer en résonance avec ses propres écrits. Inversement, un ouvrage aussi largement diffusé qu'Othea semble avoir contribué, nous le verrons, à la politisation de la figure d'Hercule vers la fin du siècle.

5 Les séquences 3 et 27 de l'Epistre Othea sont toutes deux fondées sur une matière narrative empruntée au livre $\mathrm{VII}^{16}$ de l'Ovide moralisé, l'épisode d'Hercule allant 
combattre les Infernaux pour délivrer ses compagnons d'armes, Thésée et Pirithoüs (Pirotheüs dans l'Epistre). Ce récit ne figure pas dans les Métamorphoses: c'est une addition que l'auteur français a probablement développée à partir d'une glose latine en usant très librement d'une fable qui avait son origine dans le livre VI de l'Énéide ${ }^{17}$. L'épisode de l'Ovide moralisé a pour héros principaux Thésée (à qui l'auteur attribue plusieurs des travaux d'Hercule) et son ami Pirithoüs qui, pour avoir entrepris follement de délivrer Proserpine, sont sur le point de rester prisonniers de l'enfer et d'y périr: Hercule lui-même n'intervient qu'à la fin pour ramener ses amis à la lumière. Christine n'utilise apparemment que ce seul dénouement, qui n'occupe qu'une vingtaine de vers sur deux cent vingt, mais nous verrons que de manière plus diffuse elle s'est probablement inspirée de l'ensemble de l'épisode.

6 Les deux séquences fondées sur cette fable sont d'un contenu très différent, non seulement quant à l'enseignement attaché à l'histoire, mais aussi en ce qui concerne la présentation du récit. Dans la séquence 3 en effet, qui est consacrée à la «force » dont doit faire preuve le chevalier, la déesse Othea conseille au jeune Hector de prendre modèle sur la vaillance d'Hercule, sans pourtant se croire obligé d'aller combattre en enfer ou de tenter des aventures analogues pour faire la preuve de sa valeur : l'exploit du héros est donc présenté comme un exemple tout à la fois positif et négatif. Nous reviendrons sur ce curieux détour dialectique qui contribue à la définition du contenu complexe donné au mot «force ». Notons seulement pour l'instant que dans la séquence 27, la même fable est mise à profit de manière entièrement positive pour illustrer la loyauté du chevalier qui saura affronter les périls les plus extrêmes pour secourir ses compagnons. On a pu trouver quelque contradiction dans cette façon de traiter la fable ${ }^{18}$ puisque Christine, ou Othea, en enchaînant ses enseignements, soutient d'abord que le chevalier ne doit pas tenter d'imiter cette action particulière du héros, avant d'affirmer qu'il doit « au besoin » combattre jusqu'en enfer, comme l'a fait Hercule (séq. 27, v. 5). Mais la pensée de Christine est en fait très précise et cohérente si on la rapporte, non au personnage d'Hercule, qu'il ne s'agit nullement de juger, mais à la leçon qu'il doit illustrer : aux yeux du bon chevalier, faire la guerre aux Infernaux ou aux monstres n'« est mie necessaire, /pour armes pourchacier et faire» (séq. 3, vv. 32-33), c'est-à-dire pour augmenter sa gloire; mais de telles actions deviennent un devoir «au besoing", c'est-à-dire quand il doit secourir ses compagnons. On voit ainsi comment la reprise de la même histoire, et plus généralement le morcellement du commentaire deviennent un instrument d'analyse morale.

7 Pour observer de plus près comment le matériau fourni par l'Ovide moralisé est utilisé par Christine, nous partirons du cas le plus simple, celui de la séquence 27, où Texte, Glose et Allégorie forment un développement continu sur le thème du chevalier qui «ne doit faillir a son loyal compaignon pour doubte de peril quel qu'il soit » (Glose 27): aux trois niveaux de la séquence, ce même vocabulaire est repris, pour une leçon qui est très proche des commentaires qui parsèment l'épisode de l'Ovide, à propos de l'amitié qui unit Pirithoüs et Thésée, puis ces derniers à Hercule, « qui toute sa vie / Lor avoit tenu compaignie / Bone et loial, sans fausseté » (livre VII, vv. 1935-1937). Bien que dans les deux œuvres la fable et la moralité qui y est attachée paraissent très proches, l'ensemble a en fait été remodelé par Christine pour entrer dans le cadre rigide et très strict de son Epistre. Le récit, simplifié et condensé à l'extrême, a tout d'abord été centré sur le personnage d'Hercule, devenu le seul acteur de l'histoire. Cette sorte de télescopage a conduit Christine à mettre au compte de ce nouveau protagoniste les 
actions qui dans sa source ne sont explicitement attribuées qu'à Thésée et à son compagnon. C'est le cas du coup qui brise les chaînes de Cerbère. On a parfois vu dans ce dernier détail une "erreur» de Christine : il s'agit simplement d'un effet de l'opération de concentration à laquelle elle se livre, puisque dans le récit primitif le chien infernal est libéré accidentellement par Thésée dont l'épée dévie au moment où il le frappe (Livre VII, vv. 1811-1815), alors que dans son nouveau contexte ce détail reste dépourvu de justification. Plus notable est le travail analogue effectué par Christine sur les commentaires qui accompagnent l'action dans l'Ovide moralisé. À chacune des étapes de l'aventure, en effet, le narrateur ne manque pas de souligner l'étroite et loyale amitié qui lie ses personnages: c'est elle qui décide Thésée à accepter le projet de Pirithoüs («Ne lairai, pour perdre la vie, / Que ne te tienne compaignie, / Morir en vueil, puisqu'il te plaist.» vv. 1796-1799); c'est en son nom que Pirithoüs prisonnier et en péril de mort appelle Thésée à son secours; et c'est elle enfin qui justifie l'intervention d'Hercule au dénouement. Cependant ce thème que l'Epistre Othea va ranger sous le seul nom d'Hercule, change du même coup de statut. Dans l'Ovide moralisé, en effet, il ne constitue nullement un enseignement (le seul explicitement attaché à cet épisode est d'ordre spirituel), mais plutôt une simple motivation apportée à la conduite des personnages, et plus encore un ornement amplifié à plaisir, comme il convient dans une belle histoire de chevalerie, où l'amitié se traduit en paroles éloquentes. Ainsi le traitement austère que Christine impose à son matériau de départ correspond avant tout à une nouvelle forme de moralisation, adaptée aux nécessités de ce monde et à un certain idéal de la chevalerie, même si une vérité mystique vient clore chacune des séquences. Il faut cependant observer que la valeur d'un enseignement particulier, comme celui qu'apporte la séquence 27, est largement déterminée par la perspective qu'impose l'ensemble de l'ouvrage, et à cet égard la séquence 3, où Hercule apparait pour la première fois, est une des plus éclairantes.

Cette séquence est consacrée à la troisième des quatre vertus cardinales, la « force ", qui vient après la prudence et la tempérance, et avant la justice : comme ses voisines elle est beaucoup plus développée que celles qui suivront, puisque le Texte 3 à lui seul compte quarante-cinq vers. L'ensemble de ces premières séquences forme une sorte d'ouverture qu'on peut rapprocher sur ce point de celle du De regimine principum ${ }^{19}$ et aussi des considérations liminaires du chancelier Gerson, dans son discours Vivat Rex pour la réforme du royaume (1405), sur la nécessité pour le roi de cultiver les quatre vertus cardinales, pour éviter que ne soit détruite la vie «civile et politique ${ }^{20}$. On a estimé, non sans raison, qu'elle fixait l'orientation générale de l'ouvrage: Sandra Hindman notamment y a vu la preuve que l'Epistre avait une visée politique et constituait un miroir de prince ${ }^{21}$, thèse qui a été discutée et nuancée ${ }^{22}$. Comme nous l'avons déjà indiqué, l'essentiel de la matière narrative mise en œuvre dans la séquence est emprunté à l'épisode de l'expédition en enfer longuement développé dans le livre VII de l'Ovide moralisé. Mais il est également fait allusion à d'autres exploits d'Hercule et certains détails du traitement de l'histoire, notamment le commentaire évhémériste que Christine avance dans la glose, sont peut-être inspirés, comme d'autres pages de l'Epistre, d'un chapitre très riche d'informations des Genealogie deorum gentilium libri de Boccace $^{23}$. Quoi qu'il en soit, si la brève conclusion de l'Allégorie 3 évoque la victoire sur le Malin de ceux qui sont porteurs de la parole de Dieu, l'essentiel des trois éléments de la séquence est consacré à une définition morale de la «vertu de force » telle qu'elle peut s'exercer utilement sur cette terre. 
Cet ancrage pratique, très éloigné de l'orientation générale donnée au personnage d'Hercule dans l'Ovide moralisé, est clairement souligné dans la Glose 3 au moyen de trois ensembles d'énoncés ${ }^{24}$. Tout d'abord l'accent est mis sur la définition de la «force» comme qualité morale, car elle n'est pas «seulement force corporelle, mais constance et fermeté ", si bien qu'il convient que l'exemple d'Hercule «doublement soit valable », comme modèle tout à la fois de «fais de chevalerie » et de «fort et poissant courage ». En second lieu, une explication évhémériste du mythe, qui sera reprise dans la Mutacion de Fortune $e^{25}$, met en relief le caractère historique des prouesses de ce " chevalier de Grece, de merveilleuse force ", qui ont été évoquées par les poètes «soubz couverture et en maniere de fable » lorsqu'ils « distrent que il ala en enfer combatre aux princes infernaux et que aux serpens et fieres bestes se combatoit». Enfin deux comparaisons remarquables traduisent ce qu'on pourrait appeler l'utilité publique de l'exemple apporté par Hercule: «Et ainsi comme la clarté du souleil est prouffitable a tous, peut estre bon exemple. Sicomme dit un philosophe : "Le grain de froment, quant il chet en bonne terre, il est a tous prouffitable" ${ }^{26}$." Notons surtout cette évocation d'Hercule comme personnage solaire: elle suggère un rapprochement avec Samson, dont le héros antique a souvent été au Moyen Âge une sorte de double ${ }^{27}$, comme le confirme un passage de la Mutacion de Fortune (vv. 13891-13895) qui précède le récit de la mort d'Hercule. Dans l'Advision Cristine, le nom de «Sanson le fort » sera longuement commenté comme signifiant «sol fortis" (d'après une étymologie sans doute empruntée à Isidore de Séville) et le héros biblique prendra une valeur prophétique : c'est d'un nouveau Samson que Libera, ou la France éplorée, attendra le châtiment de ses ennemis et son salut ${ }^{28}$. Si dans l'Epistre Othea les circonstances contemporaines ne sont pas explicitement évoquées (encore que certaines séquences y fassent allusion ${ }^{29}$ ), et si la présentation du personnage d'Hercule est loin d'être aussi dramatique, nous verrons que d'autres détails, dans la séquence 3, tendent également à lui donner une dimension politique.

10 L'enchaînement des commentaires qui occupent toute la Glose et la plus grande partie de l'Allégorie 3 traduit la relative homogénéité du développement consacré à la «vertu de force » représentée par Hercule. Cette longue explication correspond en fait à un approfondissement méthodique de cette notion, selon une méthode traditionnelle dans les ouvrages de philosophie morale dérivés d'Aristote : en cela elle est analogue, sous une forme réduite, à celle qui en est proposée dans la Prod'hommie de l'homme, où après une définition générale, sont énumérées «les parties ou especes d'iceste vertu " ${ }^{30}$. On peut résumer ainsi les étapes de l'analyse esquissée dans la séquence 3 de l'Epistre: la «force» est d'abord «constance et fermeté, que le bon chevalier doit avoir dans tous ses fais »; elle est également «force de resister contre les contrarietez qui lui peuent avenir, soit infortunes ou tribulacions", et plus précisément, comme il est dit dans l'Allégorie «celle qui n'est oncques brisiee en adversité et ne se orgueillist point en prosperité »; elle s'efforce d'autre part de «defendre les aournemens de vertus» de «soustenir justice»; elle est «hardie en perilz», et se montre enfin «roide contre les charneulx desirs ». Elle est donc en fait une somme de qualités qui rendent le chevalier, voire le prince, aptes à répondre à toutes les situations : elles seront définies en termes voisins dans le Livre des fais et bonnes meurs du sage roy Charles $V$ et dans le Livre du corps de policie. Le point d'aboutissement de cette réflexion sur la vertu figurée par Hercule est évidemment très éloigné parfois du personnage lui-même tel qu'il est dessiné par la 
tradition - par exemple quand il est question de la résistance aux désirs charnels - ; mais en cela Christine ne s'écarte nullement de la méthode ordinaire de l'exégèse, telle qu'elle est pratiquée par exemple dans l'Ovide moralisés ${ }^{31}$.

Nous devons cependant revenir au personnage d'Hercule et au curieux dispositif, déjà signalé, qui dès le premier élément de la séquence 3 contribue à conférer à celle-ci une portée qui dépasse la simple morale individuelle du chevalier. Sur les quarante vers du Texte initial, une trentaine est consacrée à une évocation négative de l'expédition en enfer, puis d'autres exploits du héros. Othea s'adresse en ces termes à Hector, après lui avoir proposé en exemple ses « vertus nobles et fortes »:

$[\ldots]$

Mais se tu les veulx ensuivir,

Ja pour ses vaillances suivir

Ne t'est pour tant necessaire

Aux infernaulx guerre faire

N'au dieu Pluto aler contendre

Pour Proserpine a avoir tendre

[...]

Si ne t'est mie neccessaire,

Pour armes pourchacier et faire,

Aler combatre aux fiers serpens

Aux lyons ne aux ours rampans,

Je ne sçay se tu l'imagines,

N'aussi aux autres serpentines,

Pour avoir renom de proece,

Se ce n'estoit en tel destrece

Comme pour le tien corps deffendre.

(éd. citée, pp. 204-205, vv. 12-17 et 32-40)

12 Notons l'insistance sur le conseil donné au jeune chevalier de ne pas tenter des exploits tels que ceux-ci puisqu'ils ne sont pas "nécessaires", alors qu'il lui suffit, pour prouver sa valeur et trouver la gloire, de combattre défensivement. La Glose précisera que tous ses actes devront être "deliberez par bon sens". Ces recommandations sont très proches de celles que développe Gilles de Rome à propos de la «force de courage» ou "force de vertu », qu'il présente, après Aristote, comme intermédiaire entre la peur et la témérité32 : les princes en feront preuve s'ils ne combattent que pour le bien du royaume, en évitant de s'engager par ignorance, par sottise ou par folie ${ }^{33}$. On sait combien de telles réflexions seront développées par Christine à propos des qualités de «vray chevalereux » du sage roi Charles V, dont tous les actes étaient gouvernés par la raison, le calcul, la mesure et le souci d'économiser ses forces, en un mot la prudence ${ }^{34}$. La présentation figurée de ce thème dans l'Epistre Othea, si elle tend à rapprocher l'œuvre des miroirs de prince, suggère également qu'à propos de l'exemple d'Hercule, le rapport avec l'Ovide moralisé est sans doute plus profond qu'on pourrait le penser à première vue. En effet l'aventure de Thésée et de Pirithoüs, qui tournerait au désastre sans l'intervention d'Hercule («mal bailli y eussent esté se Herculés [...] ne les eust secourus ", précise Christine dans la Glose 27, pp. 240-41), est clairement décrite comme une recherche gratuite de l'exploit. Le projet vient de Pirithoüs, qui expose à son compagnon, qu'après avoir purgé la terre de tant de monstres, ils devraient aller en enfer pour accomplir une action digne d'eux et augmenter leur gloire :

«Biaulx doulz compains, biax doulz amis,

Que j'aim plus que m'ame et mon cors,

Et vous moi tant et plus encors, 
Tant avez fait par votre effort

Qu'en cest siecle n'a riens tant fort

Qui de riens contrester vous ose.

Grans anuis est quant tant repose

Bachelers, qui pour essaucier

Son pris se doit efforcier

De biaulx fais pourchacier et querre.

Puisque nous ne trouvons sor terre

Aventure à nous esprouver,

En enfer la pourrons trouver.

Allons delivrer Proserpine [...] 》 (Livre VII, vv. 1750-1763)

Thésée voit toute la folie du projet de son ami, mais s'y rallie cependant :

«Amis, bien voi qu'amours t'afole,

Qui penser te fait tel folour.

Moult sui dolens de ta dolour

Ne lairai, pour perdre la vie,

Que ne te tiegne compaignie.

Morir en vueil, puisqu'il te plaist.

La mort o toi ne me desplaist. » (Livre VII, vv. 1794-1800)

Dans l'Ovide moralisé, cette conduite et la conception de l'esprit chevaleresque qui la détermine ne sont l'objet d'aucun commentaire moral. Mais c'est bien cette témérité insoucieuse des possibilités réelles et des conséquences de l'action, ce désir purement individualiste de la gloire que vise la séquence 3 à propos du seul Hercule, dont les autres combats se trouvent du même coup rangés allusivement dans la catégorie des exploits inutiles, à l'opposé des allégories de l'ovide, qui en font des symboles de la lutte contre le Mal. Notons que dans l'Epistre, d'autres séquences font allusion aux fautes graves commises par des chevaliers, par exemple la désobéissance d'Hector (séq. 90) ou les «folles armes» d'Ajax (séq. 94) ${ }^{35}$. Christine reprendra avec insistance sa critique de la chevalerie aventureuse, notamment dans le Livre de la Prod'hommie de l'homme (au sujet de la mesure qui n'est jamais aussi nécessaire que dans l'usage de la "force», car les chevaliers ne doivent pas "ouvrer chaudement ne a l'estourdie par oultrageuse hardiece $»^{36}$ ), et dans le Livre du corps de policie (où elle écrira que seule est digne d'éloge la hardiesse «qui est fondee sus raison et sus choses possibles et raisonnables a faire, non mie par presumpcion et fol hardement aler faire une entreprise de chose mal voirsemblable que elle puist estre tiree a bonne $\left.\operatorname{fin}^{37} »\right)$. À l'opposé, dans le Charles $V$ sont mis en avant la solidarité, l'ordre et la discipline nécessaires en pareille matière pour la défense du bien commun $^{38}$ : on sait combien ce thème d'une chevalerie réformée était d'actualité dans la situation périlleuse où se trouvait le royaume.

L'utilisation que fait Christine de l'Ovide moralisé dans la Mutacion de Fortune pour narrer l'épisode de la mort d'Hercule (VIe partie, ch. III, vv. 13885-14058) présente plusieurs analogies avec le travail opéré dans l'Epistre Othea: mais le résultat est tout à fait différent. Dans son édition de la Mutacion, Suzanne Solente place au premier rang des sources de ce récit la seconde version de la compilation intitulée par son éditeur Histoire ancienne jusqu'à César, et plus exactement le manuscrit fr. $301\left(\right.$ fol. $\left.34^{\mathrm{v}}-35^{\mathrm{r}}\right)$ de la Bibliothèque nationale de France ${ }^{39}$. Mais en réalité la fable offre dans cet ouvrage des traits qui s'écartent beaucoup de la version ovidienne reprise assez fidèlement dans la Mutacion: ainsi, pour ne donner que deux exemples qui concernent les articulations principales du récit, Déjanire, que vient d'épouser d'Hercule, est présentée dans l'Histoire comme liée à Nessus par une affection réciproque (curieux détail, peut-être 
destiné à rendre vraisemblable la confiance qu'elle accordera aux dernière paroles du centaure); en outre la femme du héros lui tend elle-même, à son lever, la chemise empoisonnée dont elle ignore le caractère funeste, si bien qu'il la prend pour la sienne dans l'obscurité matinale (selon le récit de la Mutacion et celui de l'Ovide, Hercule qui est loin de chez lui, la reçoit par un messager). En revanche la plupart des circonstances importantes du récit de l'Ovide moralisé (vv. 347-872) se retrouvent dans la Mutacion et la filiation est d'autant moins douteuse que Christine se réfère explicitement à Ovide dans la présentation du héros qu'elle place en tête de son récit et sur laquelle nous allons revenir.

Comme d'ordinaire dans cette œuvre, Christine simplifie considérablement la matière narrative qu'elle tient de sa source. Ces retranchements concernent notamment les dialogues et les monologues, souvent très longs, de l'Ovide: disparaissent ainsi les plaintes de l'épouse délaissée et les imprécations d'Hercule mourant, qui sont inutiles à l'action, tandis que d'autres éléments de ce genre, par exemple les paroles adroites que Nessus adresse à l'épouse d'Hercule, sont abrégées. Ce réaménagement, qui laisse subsister assez de détails pour que le récit reste intéressant tout en gagnant en vivacité, est dans l'ensemble assez voisin de celui que Guillaume de Machaut opère dans le Confort $\mathrm{d}^{\prime} \mathrm{Ami} \mathrm{i}^{0}$ à partir de la même source. Mais Christine tend à transformer de manière peut-être plus profonde et en tout cas cohérente les caractères de la narration. Le point de départ de ce travail est constitué par une présentation évhémériste du personnage d'Hercule (à certains égards comparable aux réflexions que Boccace lui consacre dans sa Généalogie des dieux $)^{41}$ :

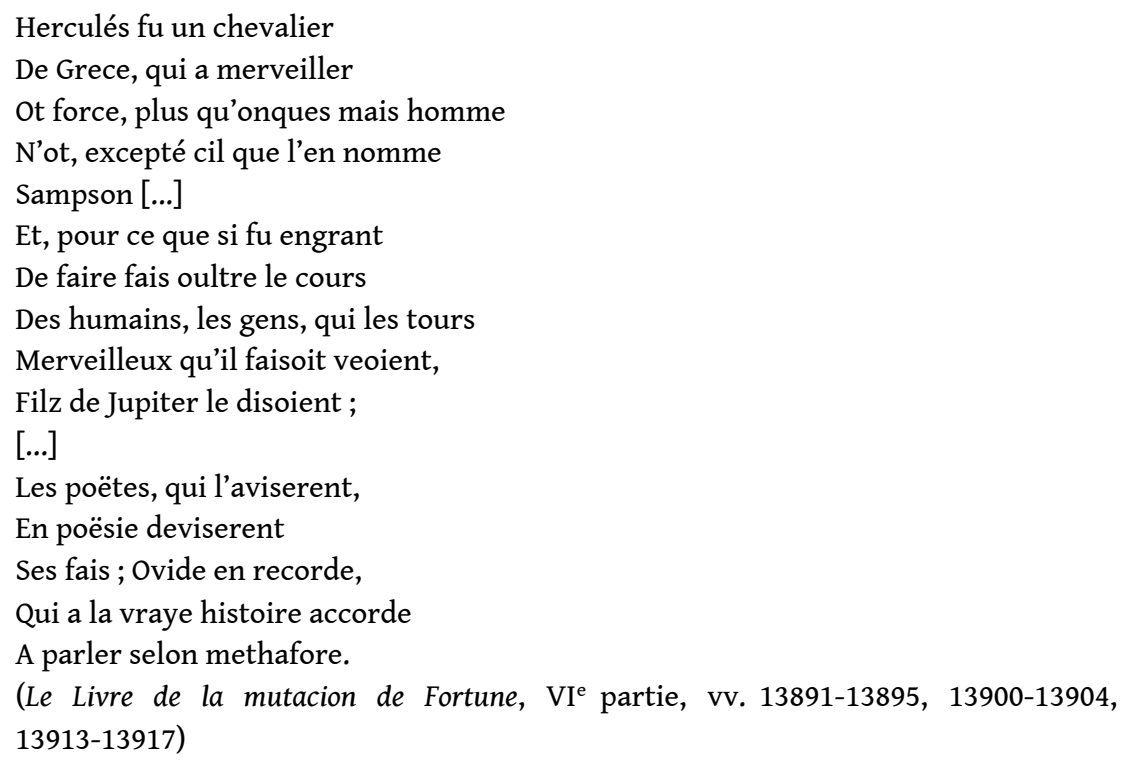

17 À la différence de l'Ovide moralisé, le récit christinien qu'introduit ce préambule va donc se situer sur le plan de la «vraye histoire»: il est supposé traduire la signification des "ymages» et de l'«escripture » que la narratrice a vues dans la salle du château de Fortune $^{42}$. Cette histoire sera logiquement allégée de la plupart des éléments proprement mythologiques ${ }^{43}$ : seuls sont conservés les éléments indispensables, car constitutifs de la fable, comme le sont l'épisode de Nessus et la chemise empoisonnée. La narration est en outre débarrassée des commentaires abondants dont elle est accompagnée dans l'Ovide moralisé, où ils constituent une partie essentielle du travail annoncé par l'auteur ${ }^{44}:$ rien ne subsiste dans la Mutacion des allégories chrétiennes qu'il développe et des réflexions morales dont il assortit les principales circonstances. Ces 
réflexions sentencieuses relèvent pour la plupart d'une tradition misogyne bien établie : la femme d'Hercule qui écoute les ultimes conseils du centaure est, comme ses semblables, écervelée et crédule ( $«$ Trop est feme legiere et fole / Et trop est muable et ventvole $\aleph^{45}$ ); amoureux, le héros est représenté avili et craintif, aux pieds d'une belle qui se plaît à l'«afoletir» (v. 557); enfin, à sa mort, son amie pleure et s'égratigne la face, mais se console vite :

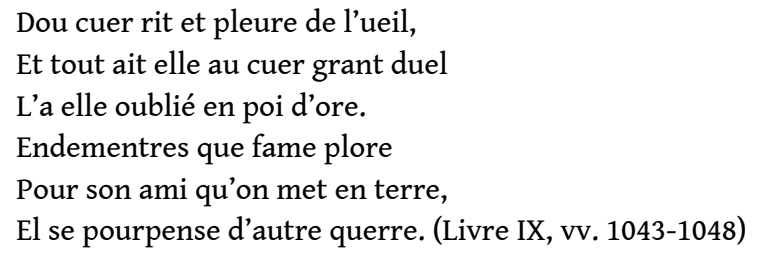

À l'opposé, la parfaite neutralité du récit de la Mutacion est d'autant plus notable qu'un épisode comme celui d'Hercule amoureux abandonnant ses armes à son amie pour se vêtir en femme appelait presque irrésistiblement, sinon un commentaire misogyne, du moins une certaine réprobation (sans y insister, Machaut écrivait cependant «Qu'il en perdi scens et memoire, / S'onneur et sa chevalerie $»^{46}$ ). Or on n'observe rien de tel de la part de Christine, et sa narration, certes très allégée en comparaison de l'Ovide moralisé, réduit de telles scènes aux proportions d'un simple jeu amoureux ${ }^{47}$. Il faut souligner d'autre part que la mort d'Hercule était traditionellement associée au procès fait aux femmes, souvent bien au-delà des réflexions fielleuses de l'Ovide moralisé. Dans le Roman de la Rose de Jean de Meung, l'épisode figure en bonne place au milieu d'une célèbre tirade sur ce thème ${ }^{48}$ : on y assure qu'Hercule, après avoir triomphé de douze monstres, ne peut triompher du treizième, Déjanire (vv. 9198-9202). Ainsi périt-il "par fame dontez», comme Samson. Eustache Deschamps, dans le Miroir du mariage, reprendra jusqu'aux termes mêmes de Jean de Meung en évoquant «Cil qui fut par femme domptez » (v. 2664) : il s'en prendra à «Dyanira la felonne » (v. 2659) qui «mit a mort Herculès le vaillant chevalier ", méritant ainsi d'être rangée auprès de Dalila, de Jézabel et de la «mauvese Thais $~_{49}$. Même si les auteurs, en reprenant ce lieu commun, gardait en fait avec lui quelque distance ${ }^{50}$, pour les lecteurs contemporains l'épisode de la mort d'Hercule devait constituer une très évidente illustration de la malfaisance féminine. Le chapitre de la Mutacion contrastait donc nécessairement avec cette attente : sa neutralité est en elle-même une réaction contre la tradition, annonçant ainsi la réhabilitation de Déjanire qui interviendra dans la Cité des dames, où elle représente, avec la châtelaine de Vergy et Yseult, celles qui «trop ont amé de grant amour sanz varier $»^{51}$. On peut s'interroger à ce propos sur l'interversion des noms féminins dans ce chapitre de la Mutacion, où Déjanire est le nom donné à la nouvelle amie d'Hercule, tandis que l'épouse qu'il délaisse est appelée Iole (v.13921). Il n'est certes pas impossible qu'il s'agisse d'une "erreur", qui est corrigée comme telle dans un manuscrit ${ }^{52}$. Mais le personnage de Déjanire était si connu que cette explication reste peu vraisemblable: cette prétendue bévue ne serait-elle pas, comme d'autres qu'ont relevées les commentateurs ${ }^{53}$. une liberté prise par l'auteur dans une intention précise? Une fois écarté ce nom, souvent rangé parmi ceux des femmes criminelles, l'épouse délaissée changeait de visage et pouvait plus aisément être considérée comme l'instrument innocent de Fortune.

L'absence de commentaires critiques concernant les actions des personnages a un autre effet, qui correspond à la finalité la plus apparente de cette disposition du récit, la mise 
en valeur de la seule responsabilité de Fortune, brièvement désignée dans la conclusion de l'épisode :

Fortune, qui moult se desguise,

Qui tant d'onneur lui ot donné,

L'a bien or en dur point mené ! (t. III, p. 24, vv. 14034-14036)

Ce n'est pourtant là qu'un rappel du leitmotiv de l'ouvrage ${ }^{54}$, et le remodelage des données empruntées à l'Ovide moralisé a une conséquence sans doute plus importante : le personnage d'Hercule s'en trouve plus clairement et vivement dessiné, si bien que les trois attributs qui le constituent dans cet épisode, la vaillance, l'amour et le malheur, y prennent un grand relief. Tous trois sont portés à un niveau superlatif, puisque l'auteur des exploits les plus extraordinaires est aussi l'amant qui pousse le plus loin l'abandon à l'amour, avant de connaître la fin la plus tragique. Ces trois éléments fondamentaux seront également à la base des événements qui occupent la longue suite de trente-deux chapitres consacrés aux guerres troyennes, immédiatement après le récit de la mort d'Hercule, comme si celui-ci préludait au thème principal de ce livre IX de la Mutacion. Cette sorte de symétrie est soulignée par la reprise, sous une forme plus développée, de la leçon tirée de la catastrophe qui a frappé le héros. Une trentaine de vers après le passage que nous avons cité (vv.14034-14036), Christine présente ainsi la nouvelle histoire qu'elle va entamer :

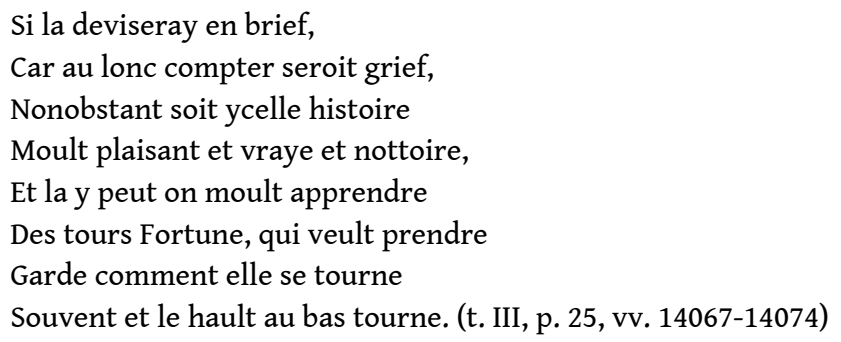

21 On voit comment à l'annonce du sens attaché au récit qui va s'ouvrir est associée cette fois l'idée du plaisir qu'y trouvera le lecteur. Comme il a déjà été dit à propos d'Hercule, ce sera une histoire illustre, vraie et du même coup instructive quant aux multiples «tours » imprévus de Fortune; mais ce sera en premier lieu, et de ce fait même, une «histoire moult plaisant»: on ne peut guère douter que cette appréciation puisse également s'appliquer à l'épisode qui vient de s'achever.

Qu'en remodelant le récit de la mort d'Hercule Christine ait surtout songé à l'agrément du lecteur peut se trouver confirmé par l'adjonction de quelques détails de son cru : ces additions étoffent quelque peu la narration et lui donnent plus de couleur à certains moments délicats. Ainsi aux cris d'effroi de l'épouse qui alertent Hercule lors de la tentative de Nessus (circonstance propre à l'Ovide moralisé et qui concorde mal avec la confiance que la jeune femme accordera à son ravisseur), Christine a substitué les moqueries du centaure qui provoquent le héros comme un mari bafoué. Plus loin elle imagine un message que l'épouse délaissée adresse à son mari en même temps que la chemise qu'elle croit devoir être bénéfique : feignant d'accepter la situation, elle lui demande du moins que "Celle chemise, qu'a ses mains / A fillee, pour l'amour d'elle / Vueille vestir $[. ..] »^{55}$. Ces additions peuvent répondre à un souci de vraisemblance, auquel s'ajoute dans le second cas un effet pathétique, puisque se trouve ainsi soulignée l'ironie cruelle de Fortune. Mais on remarquera surtout combien cette lettre imaginée par Christine donne un tour romanesque à l'histoire ${ }^{56}$. 

étudié à la fois bien adapté à des visées nouvelles, et dans chaque cas très cohérent et minutieusement conduit. Même si l'aspect le plus visible en est, dans Othea comme dans la Mutacion, une simplification des données narratives fournies par sa source et l'abandon presque complet des commentaires et allégories qu'elle y trouvait, le rapport entre ces œuvres et l'Ovide ne se réduit nullement à un simple tri : on a vu par exemple comment dans la séquence 3 d'Othea, puis de façon toute différente dans son récit de la mort d'Hercule, elle s'oppose efficacement, quoiqu'implicitement, à l'orientation idéologique du texte de départ, et du même coup à celle d'autres œuvres qui pouvaient sur ce point lui être apparentées.

Je voudrais enfin esquisser pour terminer quelques hypothèses concernant la place des œuvres de Christine dans cette "aventure d'Hercule» qui va connaître de si remarquables développements au $\mathrm{XV}^{\mathrm{e}}$ siècle. Plus d'un demi-siècle après la présentation du manuscrit de la Mutacion de Fortune à Philippe le Hardi, Raoul Lefèvre écrivait sur commande du duc de Bourgogne un Recœil des Histoires de Troyes dont Hercule était le héros principal (une version abrégée porte d'ailleurs le titre de Livre du fort Hercules ${ }^{57}$ ). Il n'est pas impossible que la fortune littéraire du héros à la cour de Bourgogne ait quelque rapport avec sa fortune politique, qui se précisera vers la fin du siècle : Hercule deviendra alors l'ancêtre mythique de la famille ducale ${ }^{58}$. Il serait certes hasardeux d'affirmer que l'œuvre de Christine ait pu jouer un rôle dans ces développements tardifs, bien que l'hypothèse ne soit pas invraisemblable, d'autant plus que les épisodes de la vie du héros évoqués dans la Mutacion se retrouveront dans la biographie romancée composée par Raoul Lefèvre. En revanche nous avons de bonnes raisons de penser que la signification politique du personnage symbolisant tous les aspects de la «force » chevaleresque et princière au début de l'Epistre Othea est restée longtemps vivace. À la fin du siècle, le héros figure dans plusieurs entrées royales, et dans l'une d'elles, produite pour l'entrée de Charles VIII à Vienne en 1490, c'est Othea, «déesse de prudence», qui tire les conclusions des histoires d'Hercule présentées au cours du spectacle ${ }^{59}$. Qu'il s'agisse de roman ou de politique, il semble en tout cas que Christine ait su en chaque occasion donner du personnage une version qui s'intégrait parfaitement à l'évolution générale des valeurs littéraires et morales, ou politiques, qui lui étaient attachées.

\section{NOTES}

1.Le Livre de la mutacion de Fortune, éd. S. Solente, 4 vol, Paris, Picard, 1959-1966, t. I, p. XXXI. Je remercie très vivement Nadia Margolis de m'avoir aidée à constituer la bibliographie de cette étude, qui ne saurait cependant prétendre à l'exhaustivité, vu l'étendue des sujets abordés.

2. «Ovide moralisé». Poème du commencement du quatorzième siècle, éd. C. De Boer, Amsterdam, N. V. Nord-Hollandsche Uitgevers-Maatschappij, 1915-1936, 5 vol. 
3.Histoire ancienne jusqu'à César (Estoires Rogier), éd. M. de Visser-van-Terwisga, Orléans, Paradigme, 1995-1999, 2 vol.

4.Epistre Othea, éd. G. Parussa, Genève, Droz, 1999 (nous nous réfèrerons à cette excellente édition); voir aussi P.G.C. Campbell, $L^{\prime}$ "Epistre Othea ». Étude sur les sources de Christine de Pizan, Paris, Champion, 1924 ; H.D. Loukopoulos, Classical mythology in the works of Christine de Pisan, with an edition of l' "Epistre Othea " from the Manuscipt Harley 4431, Ph. D., Wayne State Univ., 1977. Rappelons que l'Epistre est composée de cent « histoires» dont chacune est développée sous la forme d'une séquence de trois éléments : un «Texte » en vers, supposé prononcé par Othea, « déesse de prudence » et incarnation de la sagesse féminine, à l'intention du jeune Hector, une «Glose » et une "Allegorie ", qui se termine par une citation latine tirée des Écritures ou des Pères de l'Église. En fait la distinction entre les trois niveaux n'est pas toujours rigoureuse. 5.Ovide, Les Métamorphoses, éd. et tr. G. Lafaye, Paris, Les Belles Lettres, 1928, 3 vol. 6.Sur la conception qui préside aux commentaires : M-R. Jung, « Aspects de l'Ovide moralisé", Ovidius redivivus von Ovid zu Dante, éd. M. Picone et B. Zimmermann, Stuttgart, M \& P Verlag für Wissenschaft und Forschung, 1994, pp. 149-172, et J-Y. Tilliette, "L'Écriture et sa métamorphose. Remarques sur l'Ovide moralisé », Ensi firent li ancessor. Mélanges de philologie médiévale offerts à Marc-René Jung, éd. L. Rossi avec la coll. de Ch. Jacob-Hugon et U. Bähler, Turin, Edizione dell'Orso, 1996, 2 vol., pp. 543-558.

7.Le Livre de la mutacion de Fortune, $\mathrm{I}^{\mathrm{e}}$ partie (notamment t. I, pp. 41-46, vv. 1025-1416) ; voir K. Brownlee, «Ovide et le moi poétique "moderne” à la fin du Moyen Âge : Jean Froissart et Christine de Pizan ", Modernité au Moyen Âge : le défi du passé, éd. B. Cazelles et Ch. Méla, Genève, Droz, 1990, pp. 153-173 ; R. Blumenfeld-Kosinski, Reading myth : classical mythology and its interpretations in medieval French literature, Stanford, Standford Univ. Press, 1997, pp. 178 sq., et «Christine de Pizan and classical mythology, Some examples from the Mutacion de Fortune " The City of scholars : new approaches to Christine de Pizan, éd. M. Zimmermann \& D. De Rentiis, Berlin, New-York, de Gruyter, 1994, pp. 3-14 ; J.A. Wisman, «Christine de Pisan and Arachne's metamorphoses », Fifteenth-Century Studies 23, 1996, pp. 138-151.

8.J. Cerquiglini-Toulet, «Sexualité et politique : le mythe d'Actéon chez Christine de Pizan ", Une femme de lettres au Moyen Âge. Études autour de Christine de Pizan, éd. L. Dulac et B. Ribémont, Orléans, Paradigme, 1995, pp. 83-90.

9.K. Brownlee, «Hector and Penthesilea in the Livre de la mutacion de Fortune: Christine de Pizan and the politics of myth », Une femme de lettres au Moyen Âge, op. cit., pp. 69-82. 10.Éd. cit., pp. 76 sq. 11.J. Seznec, La Survivance des dieux antiques : essai sur le rôle de la tradition mythologique dans l'humanisme et dans l'art de la Renaissance, Paris, Flammarion, 1993, dont les vues sont critiquées dans un article important de F. Gaeta, "L'avventura di Ercole ", Rinascimento 5, 1954, pp. 227-260 ; voir aussi M-R. Jung, Hercule dans la littérature française du XVI ${ }^{e}$ siècle, Genève, Droz, 1966.

12.Gaeta, art. cit., p. 233.

13.Les travaux d'Hercule et son apothéose occupent la plus grande partie de la conclusion en vers (chant VII) du livre IV (Boèce, Philosophiae Consolacio, éd. L. Bieler, Turnhout, Brepols, 1957, pp. 87-88) ; voir G.M. Cropp, « Boèce et Christine de Pizan », Le Moyen Âge, 3-4, 1981, pp. 387-. 417, et G.M. Cropp, "The figure of Hercules in the medieval French translations of the Consolatio Philosophiae, "Variété: Perspectives in 
French literature, society and culture ", éd. M. Ramsland, Francfort, Peter Lang, 1999, pp. 67-76.

14.Gaeta, art. cit., pp. 242 sq. ; également M. Simon, Hercule et le christianisme, Paris, Belles lettres, 1955, pp. 49-50.

15.Jung, Hercule, op. cit., pp. 38 sq.

16.Éd. cit., t. III, livre VII, vv. 1730-1951 pour le récit lui-même, qui est commenté ensuite jusqu'au v. 2068.

17.Énéide, VI, vv. 392-397, 601, 617-618; sur cette addition : P. Demats, Fabula. Trois études de mythographie antique et médiévale, Genève, Droz, 1973, pp. 63-65.

18.Cropp, «The Figure of Hercules », art. cit., p. 74.

19.Gilles de Rome, Li Livres du gouvernement des rois. A XIII ${ }^{\text {th }}$ Century French version of Egidio Colonna's treatise «De regimine principum», éd. S. P. Molenaer, New York-Londres, 1899, pp. 35 sq.

20.Jean Gerson, CEuvres complètes, éd. P. Glorieux, Paris-Tournai, Desclée, 1960-1973, 10 vol., t. 1 VII, 2, L'Euvre française, Sermons et discours, p. 1149.

21.S.L. Hindeman, Christine de Pizan's «Epistre Othea ». Painting and politics at the court of Charles VI, Toronto, Pontifical Institute of Medieval Studies, 1986, pp. 51-55.

22.Voir par exemple E. Carrara, « Mitologia antica in un trattato didattico-allegorico della fine del Medioevo : L'Epistre Othea di Christine de Pizan », Prospettiva, 66, 1992, pp. 67-86 (notamment p. 83, où sont reprises des observations de G. Mombello parues dans Studi francesi 95, 1988, pp. 315-316). G. Parussa écrit à ce sujet : « Nous ne pensons pas [...] que l'Epistre ait été conçue, selon la volonté de l'auteur, comme un véritable miroir de prince [...] Ce que nous tenons à souligner c'est la présence de caractéristiques thématiques et formelles qui permettent d'établir un lien entre l'Epistre et les miroirs [...].» (G. Parussa, «Instruire les chevaliers et conseiller les princes : L'Epistre Othea de Christine de Pizan ", Studi di storia della civiltà letteraria francese. Mélanges offerts à Lionello Sozzi, Paris, Champion, 1996, vol. I, pp. 129-155, ici pp. 148-149). Sur les rapports entre les œuvres de Christine et les écrits politiques de son temps, voir J. Blanchard, J-C. Mühlethaler, Écriture et pouvoir à l'aube des temps modernes, Paris, PUF, 2002, pp. 18-26, et J-C. Mühlethaler, «Traictier de vertu au proufit d'ordre de vivre : relire l'œuvre de Christine de Pizan à la lumière des miroirs des princes ", Contexts and Continuities. :Proceedings of the IVth international colloquium on Christine de Pizan (Glasgow, 21-27 juillet 2000), éd. A. Kennedy, with R. Brown-Grant, J.C. Laidlaw \& C. Müller, Glasgow Univ. Press, 2002, pp. 585-601.

23.G. Boccace, Genealogie deorum gentilium libri, éd. V. Romano, Bari, G. Laterza \& Figli, 1951, 2 vol., t. II, pp. 632-643 (livre XIII, ch. I) et Epistre Othea, p. 390.

24. Epistre Othea, pp. 205-206.

25.Le Livre de la mutacion de Fortune, $\mathrm{VI}^{\mathrm{e}}$ partie, vv. 13891-13918 (t. III, pp. 19-20).

26. Epistre Othea, Glose 3, pp. 205-206.

27. Hercule et Samson sont par exemple associés dans l'Histoire ancienne jusqu'à César (éd. cit., t. I, p. 82, § 138 et t. II, p. 155, notes), dans le Roman de la Rose (Guillaume de Lorris et Jean de Meun, Le Roman de la Rose, éd. A. Strubel, Paris, Le Livre de poche, 1992, p. 548, vv. 9187 sq.) ou encore dans le Miroir de mariage d'Eustache Deschamps (Euvres complètes, éd. Marquis de Queux de Saint-Hilaire et G. Raynaud, Paris, F. Didot, 1878-1903, 11 vol., t. IX, p. 181, vv. 5529-5534).

28. Le Livre de l'advision Cristine, éd. Ch. Reno et L. Dulac, Paris, Champion, 2001, pp. 36-37. 
29.G. Parussa en donne plusieurs exemples dans «Instruire les chevaliers [...] , art. cit. p. 146 : «Derrière les références toutes traditionnelles à la littérature morale et didactique médiévale, on ressent toutefois le poids des événements du temps [...]». 30.Le Livre de la Prod'hommie de l'homme (ou Le Livre de Prudence), éd. E. Hicks et S. Pagot (en préparation), dans la copie obligeamment communiquée par E. Hicks, d'après le ms Biblioteca apostolica vaticana, Reg. lat. 1238, fol. 42r-42v.

31.R. Blumenfeld-Kosinski, Reading myth, op. cit., pp. 90 sq., et H. Campangne, Mythologie et rhétorique aux $X^{e}$ et $X V I^{e}$ siècles en France, Paris, Champion, 1996, pp. 50 sq.

32.Éd. cit., pp. 49-50.

33.Éd. cit., pp. 53-54.

34.Le Livre des fais et bonnes meurs du sage roy Charles V, éd. S. Solente, Paris, Champion, 1936-1940, 2 vol. (réimpr. Genève, Slatkine-Mégariotis, 1977), t. I, pp. 116 sq.

35.J.K. Kellog, "Christine de Pizan as chivalric mythographer : L'Epistre Othea », The Mythographic art : classical fable and the rise of the vernacular in early France and England, éd. J. Chance, Gainesville, Univ. of Florida Press, 1990, pp. 100-124 (à ce propos pp. 110-111). 36.Ms cité, fol. $37^{\mathrm{v}}-38^{\mathrm{r}}$.

37.Le Livre du corps de policie, p. 65, 11. 27-30.

38. Le Livre des fais et bonnes meurs du sage roy Charles V, éd. cit., t. I, pp. 116-120. Jacques Krynen rappelle qu'aux yeux des clercs, dès l'époque des premières défaites de la guerre de Cent Ans, «c'est moins de prouesses dont la chevalerie a besoin que d'une discipline spécifique : une disciplina militaris»; cependant « toute une littérature entretient le public nobiliaire dans une conception traditionnelle de la guerre, joyeuse et individualiste» (L'Empire du roi. Idées et croyances politiques en France. XIII ${ }^{e}$-XIVe siècle, Paris, Gallimard, 1993, pp. 322-323). Voir également J. Blanchard, J-C. Mühlethaler, Écriture et pouvoir, op. cit., pp. 109-111.

39.Le Livre de la mutacion de Fortune, éd. cit., t. I, pp. LXIII sq., et à propos de la mort d'Hercule, t. III, p. 274.

40.Guillaume de Machaut, Le Confort d'ami, vv. 2683-2784, dans CEuvres, éd. E. Hoepffner, Paris, F. Didot, puis Champion, 1908-1921, 3 vol., t. III, pp. 95-97.

41.G. Boccace, Genealogie deorum gentilium libri, op. cit., livre XIII, ch. I, pp. 639 sq. 42.Début de la IV e partie, éd. cit., p. 99 sq. (vv. 7053-7172) ; voir aussi les vv. 14059-14066 qui précèdent les récits des guerres de Troie. Sur l'attitude de la narratrice et son " détachement ", voir N. Margolis, "Christine de Pizan : the poetess as Historian », Journal of the History of Ideas, XLVII, 3, 1986, pp. 361-375, et « The Rhetoric of detachment in Christine de Pizans's Mutacion de Fortune ", Nottingham French Studies, vol. 38, 2, Autumn 1999, pp. 170-181; K. Brownlee, «The Image of History in Christine de Pizan's Livre de la Mutacion de Fortune ", Yale French Studies, (Special Issue), Contexts, Style and values in medieval art and literature, éd. D. Poirion \& N.F. Regalado, 1991, pp. 44-56. 43. Ce traitement peut être rapproché de celui qui historicise les mythes antiques dans des œuvres comme le Roman de Troie de Benoît de Sainte-Maure : voir E. Baumgartner, «Remarques sur la réception des mythes antiques dans la littérature française du XII au XIII ${ }^{\mathrm{e}}$ siècle », L'Antiquité dans la littérature européenne du Moyen Âge, Greifswald, Reineke-Verlag, 1998, pp. 135-148.

44. Voir le prologue de l'ovide moralisé : l'auteur y marque bien son ambition de mettre au jour, plus complètement que ses prédécesseurs, les vérités cachées dans «les fables 
de l'ancien temps » (éd. cit., t. I, pp. 61-62); cf. H. Campangne, Mythologie et rhétorique, op. cit., pp. 50-54.

45.0vide moralisé, éd. cit., livre IX, vv. 439-440.

46.Le Confort d'Ami, éd. cit., vv. 2732-2733.

47. Même présentation positive de l'épisode de la part de Marote dans le Dit de la pastoure (Euvres poétiques de Christine de Pisan, éd. M. Roy, Paris, Didot, 1886-1896, réimpr. Johnson reprint Corp., 1965, 3 vol., t. 2, pp. 268-269, vv. 1459-1477) ; cf. E. Benkov, «Unmanning Hercules : Myth and gender in the Dit de la pastoure », Contexts and continuities, op. cit., t. I, pp. 65-74.

48.Le Roman de la Rose, éd. cit., vv. 9043 sq., pp. 540-549.

49. Le Miroir de mariage, XXIX, vv. 2648-2667, CEuvres complètes, éd. cit., t. IX, pp. 89-90.

50.A. Strubel dans Le Roman de la Rose, éd. cit., pp. 27-28.

51.Le Livre de la Cité des dames, livre II, ch. LX, dans La Città delle Dame, éd. E.J. Richards, intro. et tr. italienne P. Caraffi, Milan, Luni Editrice, 1998, p. 402. Sur ces questions, voir R. Brown-Grant, Christine de Pizan and the moral defence of women. Reading beyond gender, Cambridge, Cambridge University Press, 1999.

52.Le Livre de la mutacion de Fortune, éd. cit., t. III, v. 13921, 13984, 13999 (corrections portées sur le ms E).

53.J.S. Holderness, "Christine et ses “bévues” : sens et portée de quelques assimilations abusives ", Au champ des escriptures, III Colloque international sur Christine de Pizan, Lausanne, 18-22 juillet 1998, éd. E. Hicks, avec la coll. de D. Gonzalez et P. Simon, Paris, Champion, 2000, pp. 149-160.

54. Chaucer, après avoir évoqué la mort d'Hercule dans Le conte du moine, en fait également un exemple des coups imprévus de Fortune (Geoffrey Chaucer, Les Contes de Canterbury, tr. André Crépin, Paris, Gallimard, 2000, pp. 493-494).

55.Vv. 14010-14012.

56.On sait combien la lettre est un objet romanesque : en témoigne sa place, par exemple, dans le Tristan et Yseult de Béroul (vv. 2336 sq., 2640 sq., etc.) ou dans Le Roman du comte d'Anjou de Jehan Maillart (éd. M. Roques, Paris, Champion, 1964, vv. 3574 sq., 4833 sq.).

57.Raoul Lefèvre, Le Recœil des Histoires de Troyes, éd. M. Aeschbach, Berne, Francfort, Paris, P. Lang, 1987 (Livres I et II) ; analyse dans Jung, Hercule, op. cit., pp. 16-26.

58.J. Seznec, La Survivance des dieux antiques, op. cit., p. 36.

59.Jung, Hercule, op. cit., pp. 38-39.

\section{AUTEUR}

\section{LILIANE DULAC}

Université de Montpellier III 